\title{
EL TRASTORNO POR DÉFICIT DE ATENCIÓN E HIPERACTIVIDAD (TDAH), DIFERENCIAS ENTRE LOS DIFE- RENTES SUBTIPOS EN LA COMPOSICIÓN ESCRITA
}

\author{
Jesús Nicasio Garcia-Sánchez \\ Celestino Rodríguez-Párez \\ Ana $M^{a}$ de Caso-Fuerte \\ Raquel Fidalgo-Redondo \\ Olga Arias-Gundín \\ Lorena González-Sánchez \\ Begoña Martínez-Cocó
}

Universỉdad de León. Departamento de Psicologla, Sociologla y Filosofia. Area de Psicologla Evolutiva y de la Educación

\section{RESUMEN}

Se presentan datos epidemiológicos detectados de una muestra de alumnos de primaria y secundaria $(N=1004)$ procedentes de 24 centros educativos de la provincia de León, $y$ que han sido estudiados en base a los procesos $y$ factores psicológicos de la escritura. Los datos indican que un $26(2,59$ $\%)$ han sido preseleccionados por los maestros y profesores como posibles TDAH con el cuestionario DSM-IV. $22(2,29 \%)$ pudieron clasificarse en el subtipo de problemas atencionales,

Durante la realización de esta investigación se recibieron ayudas de la JCyL, para 20062008 (LE028A06), proyecto de investigación competlivo concedido al IP, primer autor: Solapamiento y diferenciación de los problemas atencionales y de hiperactividad e impulsividad en base a la composición escrita (procesos y factores psicológicos), en alumnos con y sin diffcultades de aprendizaje.

Correspondencia: Jesús-Nicasio Garcla-Sánchez, Universidad de León, Departamento de Psicologia, Sociologla y Filosofla, Area de Psicologla Evolutiva y de la Educación, Campus de Vegazana. s/n, 24071-León, España, tf.: +34-987 2910 41; +34-652 8178 71; E-mail: jn.garcia@unileon.es. 
$13(1,29 \%)$ de problemas de hiperactividad e impulsividad y 19 $(1,89 \%)$ en el subtipo combinado. A la par, se diferencian estos subtipos en muchas de las medidas de composición escrita. Se valoran y discuten las implicaciones educativas y teóricas.

Palabras clave: TDAH, COMPOSICIÓN ESCRITA, SUBTIPO INATENTO, SUBTIPOHIPERACTIVOMMPULSIVO, SUBTIPO COMBNADO, CUESTIONARIODSM-N

\section{SUMMARY}

Epidemiological data extracted from a wide sample of Primary and Secondary School students $(N=1004)$ who received education in 24 Schools from the Leon province (Spain). They were studied with the focus in writing psychological factors and processes. 26 students $(2,5 \%)$ were preselected by the teachers as having a possible $A D H D$ with the DSM-IV questionnaire. $A$ part of this subsample, 22 students $(2,29 \%)$ could be classified in the subtype of attention disorder, 13 students $(1,29 \%)$ in the subtype of hyperactivity and impulsivity disorder, and 19 students $(1,89 \%)$ would be classified into the combined subtype. Besides, it was possible to distinguish those subtypes in different writing composition measures. We appreciate and discuss the theoretical and educational implications.

Koy words: ADHD, WRITING COMPOSITION, INATTENTIVE SUBTYPE, HYPERACTIVE-IMPULSIVE SUBTYPE, COMBINED SUBTYPE, DSM-N QUESTIONNARE

\section{INTRODUCCIÓN}

Se presenta un estudio de detección de alumnos con problemas atencionales, de hiperactividad y/o de impulsividad, dentro de un proyecto más amplio de desarrollo de la composición escrita desde perspectivas psicológicas e instruccionales, que persigue implementar instrumentos de evaluación de los procesos y factores psicológicos 
que participan en la composición escrita así como el diseño y aplicación de intervenciones de optimización de los mismos con alumnos con y sin DA. Desde esta perspectiva, tres son las cuestiones que se intentan responder. En primer lugar, ¿cuántos casos de TDAH hay (prevalencia) en función del criterio de los profesores en una muestra masiva de la provincia de León?, ¿coinciden o no con los datos epidemiológicos de otros estudios o con los que proporciona la DSM-IV? En segundo lugar, ¿se diferencian estos problemas y por subtipos en base a los niveles de redacción (productividad y coherencia) o en otras variables? Y por último, ¿qué implicaciones podemos sacar de estos resultados, a nivel educativo o a nivel teórico en relación con los problemas que presentan en composición escrita las personas con TDAH?

Los problemas del TDAH, que incluyen inatención, hiperactividad e impulsividad, han sufrido diferentes conceptualizaciones a lo largo de la historia (Barkley, 1997 a; b; 1998; 2001; Collings, 2003). Estos problemas son muy frecuentes, ya que ocurren entre el $3 \%$ y el $6 \%$ de niños en edad escolar (APA., 1994) y persisten en los adultos en el $40 \%$ de los casos (Klein \& Manuzza, 1991) tal y como refiere la revisión de Nolan et al., (1999). Debido a la frecuencia de este problema, la sociedad debe preocuparse y atender a las personas con estas dificultades, a través de la creación de servicios educativos, clínicos y sociales.

Una idea importante que se observa, es el solapamiento del TDAH con otras dificultades o trastornos. Por ejemplo, se ha descrito el $80 \%$ de solapamiento con 2, 3 o más trastornos del desarrollo (Kaplan, Dewey, Crawford, \& Wilson, 2001). Sin embargo, los estudios sobre el solapamiento son escasos y en español, prácticamente inexistentes al igual que los referidos a las dificultades en composición escrita. Este será el núcleo de nuestra investigación.

Dada la alta prevalencia y el alto solapamiento, y dado que las personas con el TDAH presentan un déficit en el control de impulsos, es probable que esto se refleje en la composición escrita. Sabemos que escribir implica reflexionar sobre el propósito, la audiencia, los elementos retóricos, el esquema, los detalles, la complejidad, los resultados, la coherencia, etc., (Alamargot \& Chanquoy, 2001; AriasGundIn \& Garcia, 2006; 2007; Garcla \& de Caso, 2002, 2006; Kellogg, 1994; Torrance \& Galbraith, 1999; Wong, 2000). 
Desde esta reflexión surge una doble idea, la primera es si las dificultades en la escritura son problemas secundarios al TDAH o son problemas comórbidos al TDAH y la segunda tiene que ver con la forma de intervenir sobre estas dificultades.

En este sentido, el escritor efectivo, selecciona, adopta o inventa estrategias que mejoren sus objetivos (Buttler, Elaschuk \& Poole, 2000; de la Paz, 1999; 2001), es decir, de forma autorregulada (Fidalgo, Torrance, \& García, en prensa; García \& Fidalgo, 2003, en prensa; Graham \& Harris, 2000; 2002; Torrance, Fidalgo, \& García, 2007), lo que puede suponer el uso de un estilo cognitivo eficiente, como el reflexivo. La intervención focalizada en la mejora de estrategias de procesamiento reflexivo en tareas de composición escrita parece de interés (Garcla \& de Caso, 2002, 2007), así como en los casos de solapamiento entre TDAH y problemas en la composición escrita

Hemos de considerar que el esfuerzo mental y los recursos atencionales son limitados cuando se realizan tareas de escritura (Kellogg, 1994; Alamargot \& Chanquoy, 2001). Esto implica que han de considerarse a la vez muchos procesos (la búsqueda de la palabra o el grafema, el tema, las ideas, la audiencia o el plan general de la composición escrita) y de forma autorregulada y evolutiva (Garcia \& Fidalgo, 2003); por lo que, si hubiera dificultad atencional, ello se reflejaría en la composición escrita y en el complejo proceso de escribir (Gregg, Coleman, Stennett, \& Davis, 2002).

Parece relevante el estudio del solapamiento desde el punto de vista evaluativo y de la intervención, centrándose en la contextualización y los multicomponentes y considerando que el problema nuclear está en el déficit de la auto-regulación y el control de impulsos (Barkley 1997 a; b; 1998 ;2001; Miranda \& Presentación, 2000) y ello hacerlo en relación con la escritura. Por ejemplo, desde la teoría de Barkley (1997 a; b), el problema que presentan las personas con TDAH es un déficit en la inhibición comportamental y que implica a cuatro grandes funciones ejecutivas (memoria de trabajo; autoregulación del afecto/ motivación/activación; internalización del lenguaje; y reconstitución); todo ello afectando al control motor/fluencia/sintaxis de la conducta. Es previsible que desde el estudio de la composición escrita se aporten algunas luces para la comprensión del problema que presentan estas personas. 
Diferentes estudios demuestran la existencia del solapamiento del TDAH con otros problemas. Asi, Taylor, (1995) determina que el $70 \%$ de niños con TDAH presentan DA, siendo el TDAH el trastorno que prevalece.

El solapamiento lo respaldan estudios como el de Kaplan et al., (2001) en el que se dice que los niños con TDAH tienen un alto riesgo de tener una segunda dificultad. Asimismo, los niños con déficit de atención tienen una alta prevalencia en problemas del lenguaje. En este estudio 63 de los 126 niños con dificultades en la lectura, fueron diagnosticados con TDAH; 47 de los 63 que se diagnosticaron con TDAH tenían una dificultad más añadida, 30 tenían 2 y 13 tenían 3 . Si el niño tenía dificultades en la lectura la posibilidad de tener otro trastomo era del $51,6 \%$, sin embargo si tenia TDAH la posibilidad era del $80,4 \%$.

Se estima que entre el $19 \%$ y el $26 \%$ de personas con TDAH tienen algún tipo de DA, lo que evidencia el papel de los problemas atencionales en el ámbito escolar (Barkley, 1996)

La mitad de los 1,6 millones de escolares diagnosticados con TDAH y con problemas de atención fueron identificados con otra dificultad de aprendizaje, según el centro de control y prevención, CDC (NHIS, 1997-1998).

Otros estudios como el de Willcutt y Pennington, (2000) indican que los niños con problemas de TDAH y con criterios de déficit atencional (DA), aparte de solaparse ambos problemas frecuentemente, presentan una historia de la familia, de DA o procesamiento cognitivo lento.

En otro estudio, niños con DA y bajo rendimiento han dado déficits en estrategias de autorregulación en las composiciones escritas y de la capacidad de reflexión en la realización de las mismas (Graham \& Harris, 1999).

Según Tabassam y Grainger (2002), 1/3 de los niños que tienen DA presentan a su vez algún subtipo del TDAH.

Miranda y Presentación (2000) apuntan que alumnos con DA y bajo rendimiento se relacionan con una menor reflexividad y presentan un alto solapamiento con problemas de impulsividad. Por último, Kaplan et al., (2001) señalan en su estudio que se encuentra una alta coincidencia (comorbilidad), de problemas del desarrollo y del aprendizaje. 
Estos estudios muestran la necesidad y la relevancia de continuar y profundizar en la investigación sobre el solapamiento entre el TDAH y las DA, ya que muy pocos han intentado identificar las relaciones entre subtipos frecuentes, en alumnos con TDAH y DA, existiendo pues estudios escasos (Gregg et al., 2002). Se necesitan investigaciones más especificas y más profundas. Además, faltan estudios que consideren el significado educacional del solapamiento entre el TDAH y los problemas en la composición escrita.

\section{MÉTODO}

\section{Participantes}

En el presente estudio participaron 1.004 alumnos de 24 centros públicos de la provincia de León, pertenecientes a los cuatro CEFIEs de la misma (Astorga, León, Ponferrada y Villablino). Todos los alumnos perteneclan al último ciclo de Educación Primaria $\left(5^{\circ}\right.$ y $\left.6^{\circ}\right)$ y al primer ciclo de Educación Secundaria Obligatoria $\left(1^{\circ}\right.$ y $\left.2^{\circ}\right)$, y sus edades estaban comprendidas entre los 9 y los 15 años.

\section{Instrumentos}

Dentro de la aplicación colectiva del Instrumento de Revisión de la Composición Escrita (IRCE) (Arias \& Garcla, 2003, ver tamblén Arias \& Garcia, 2006, 2007), se utilizó en este articulo, únicamente el análisis de la tarea de redacción, primera prueba que realizan los alumnos al implementarse dicho instrumento. En esta tarea se evalúa la productividad, en términos de cantidad de texto producido y la calidad del texto escrito, referida a la coherencia textual. La productividad o cantidad de texto elaborado por el alurnno, entendida como generación de información, se comprueba a través de los siguientes parámetros: oraciones, verbos en formas personales, palabras de contenido, palabras funcionales y determinantes. Por otro lado, la calidad del texto, entendiéndose ésta como la presentación organizada de la información elaborada, se estudió a través de la coherencia referencial, la coherencia relacional, el empleo de ideas pertinentes, la utilización correcta de los enlaces, la delimitación adecuada de los párrafos 
coherentemente estructurados y el desarrollo del hilo argumental de todo el texto.

Además, se solicitaba de los profesores y maestros que identificaran a los alumnos que dentro de sus clases consideraran con problemas de desatención, de hiperactividad o de impulsividad. En este caso, cumplimentaban el cuestionario DSM-IV para profesores, que recoge en forma escalar (de 1 a 4) cada uno de los criterios diagnósticos para el trastomo de atención ( 9 ítems) o para el trastorno hiperactivo impulsivo ( 9 ítems). Teniendo en cuenta que para considerar problema atencional (TDAH-A) ha de haber seis o más items puntuados con 30 más, se tomó como punto de corte 21 (sobre un total posible de 36), y lo mismo se hizo para el caso de problemas hiperactivo-impulsivos (TDAH-HI). Para el caso del subtipo combinado, se exigía un punto de corte de 42 sobre un total posible de 72.

\section{Procedimiento}

Durante el mes de septiembre se realizaron los contactos pertinentes con los centros para la obtención de los permisos oportunos, así como determinar las fechas para realizar la implementación de la bateria de evaluación. A lo largo de los meses de octubre y noviembre del curso escolar 2003-2004, se aplicó de forma colectiva el IRCE a los 50 grupos participantes en el proceso de validación del mismo. Con este instrumento se tomaron además de las medidas de productividad y coherencia que aquí se presentan, medidas de los diferentes componentes relacionados con el proceso de revisión como son el nivel textual donde se produce, la localización de la misma, así como las estrategias seleccionadas y ejecutadas. Una vez concluida la corrección de la tarea de redacción llevada a cabo por siete evaluadores, las medidas fueron codificadas y sometidas a análisis estadistico con el programa SPSS versión 11.01. E, igualmente se hizo con los datos del cuestionario DSM-IV para profesores.

\section{RESULTADOS}

Prevalencia de los problemas atencionales, de hiperactividad e impulsividad 
De un total de 1004 alumnos estudiados, los profesores y maestros identificaron 26 posibles casos. Tras la cumplimentación del cuestionario DSM-IV para el TDAH se conflrmaron 19 casos que cumplian los criterios del subtipo combinado o el 1,98\%, con seis ítems $\delta$ más en desatención y otros tantos en hiperactividad e impulsividad; 13 casos que cumplieran los criterios hiperactivoimpulsivos $(1,29 \%)$; y 22 casos del subtipo desatento $(2,291 \%)$.

Diferencias por subtipos en base a niveles en redacción

Cuando se analizan las diferencias en niveles de redacción y otras variables en función de los subtipos comparados con los alumnos sin estos problemas, encontramos diferencias estadísticamente significativas en varias variables.

Cuando se comparan los alumnos del subtipo combinado (TDAHC) con los normales, mediante ANOVAS, se encuentran diferencias estadísticamente significativas en generación de ideas en número de oraciones con una media del subtipo combinado $\left[M_{c}=3,22\right]$ inferior a la de los normales $\left[M_{N}=6 ; F_{(1,999)}=12,94 ; p=0,00\right]$; número de verbos en forma personal $\left[M_{c}=10,77\right.$ frente a $M_{N}=16,12 ; F_{(1,999)}=$ $8,62 ; \mathrm{p}=0,00]$; número de palabras de contenido $\left[\mathrm{M}_{\mathrm{c}}=33,77\right.$ frente a $\left.M_{N}=48,28 ; F_{(1,999)}=9,90 ; p=0,00\right]$; número de palabras funcionales $\left[M_{c}=23,27\right.$ frente a $\left.M_{N}=31,45 ; F_{(1,999)}=4,00 ; p=0,04\right]$; número de determinantes $\left[M_{c}=11,55\right.$ frente $a M_{N}=15,55 ; F_{(1.999)}=4,37 ; p=$ $0,03]$; en hilo argumental $\left[\mathrm{M}_{C}=0,5\right.$ frente a $\mathrm{M}_{N}=0,76 ; \mathrm{F}_{(1,999)}=6,62$; $\mathrm{p}=0,01]$; en coherencia total $\left[\mathrm{M}_{\mathrm{c}}=0,77\right.$ frente a $\mathrm{M}_{\mathrm{N}}=2,61 ; \mathrm{F}{ }_{(1,999)}=$ $29,61 ; p=0,00]$; número de comas, punto $y$ aparte $y$ dos puntos marginalmente significativo $\left[M_{c}=3\right.$ frente a $M_{N}=4,96 ; F_{(1,999)}=3,16$; $p=0,07]$; número de puntos y seguidos $\left[M_{c}=3,22\right.$ frente a $M_{N}=5,72$; $\left.F_{(1,999)}=11,12 ; p=0,00\right]$; en la edad $\left[M_{C}=12,31\right.$ frente a $M_{N}=11,71$; $\left.F_{(1,999)}=3,64 ; p=0,05\right]$; y en la nota aportada por el alumno del último trimestre en lengua $\left[M_{c}=4,16\right.$ frente $a M_{N}=5,58 ; F_{(1,999)}=5,15 ; p=$ 0,02].

Para el caso de los alumnos con el subtipo hiperactivo-impulsivo (TDAH-HI) en comparación con los normales, mediante ANOVAS, se encuentran diferencias estadisticamente significativas en tiempo (próximo a la significación) mayor en los hiperactivo-impulsivos $\left[\mathrm{M}_{\mathrm{c}}=\right.$ 84,61 frente $\left.a M_{N}=79,99 ; F_{(1,999)}=3,4 ; p=0,06\right]$; generación de 
ideas en número de oraciones con una media del subtipo hiperactivoimpulsivo $\left[M_{H 1}=3,16\right]$ inferior a la de los normales $\left[M_{N}=5,98 ; F\right.$ $=8,89 ; p=0,00]$; número de verbos en forma personal $\left[\mathrm{M}_{\mathrm{HI}}=11,33\right.$ frente a $\left.M_{N}=16,08 ; F_{(1,999)}=4,55 ; p=0,03\right]$; número de palabras de contenido $\left[M_{H 1}=36,41\right.$ frente a $\left.M_{N}=48,16 ; F_{(1,999)}=4,33 ; p=0,03\right]$; en hilo argumental $\left[\mathrm{M}_{\mathrm{H}}=0,5\right.$ frente a $\mathrm{M}_{\mathrm{N}}=0,76 ; \mathrm{F}_{(1, \text { g99 }}=4,38 ; p=$ $0,03]$; en coherencia total $\left[M_{c}=0,76\right.$ frente a $M_{N}=2,75 ; F_{(1,999)}=$ 22,77; $p=0,00]$; y en número de puntos y seguidos $\left[\mathrm{M}_{\mathrm{H1}}=3,16\right.$ frente a $\left.M_{N}=5,71 ; F_{(1,999)}=7,68 ; p=0,00\right]$.

$Y$ por ültimo, cuando se comparan los alumnos del subtipo inatento (TDAH-A) con los normales, mediante ANOVAS, se encuentran diferencias estadísticamente significativas en generación de ideas, número de párrafos con una media del subtipo inatento $\left[\mathrm{M}_{\mathrm{A}}\right.$ $=1,77]$ inferior a la de los normales $\left[M_{N}=2,56 ; F_{(1,999)}=3,74 ; p=\right.$ $0,05]$; en número de oraciones $\left[\mathrm{M}_{\mathrm{A}}=3,45\right.$ frente $a \mathrm{M}_{\mathrm{N}}=6 ; \mathrm{F}_{(1,999)}=$ $13,29 ; p=0,00]$; en número de verbos en forma personal $\left[M_{A}=10,81\right.$ frente $\left.a M_{N}=16,14 ; F_{(1,999)}=10,44 ; p=0,00\right]$; en número de palabras de contenldo $\left[\mathrm{M}_{A}=32,77\right.$ frente a $M_{N}=48,36 ; F_{(1, \text { g99) }}=14,07 ; p=$ $0,00]$; en número de palabras funcionales $\left[\mathrm{M}_{A}=23,22\right.$ frente a $\mathrm{M}_{N}=$ 31,$\left.48 ; F_{(1,999)}=4,98 ; p=0,02\right]$; en número de determinantes $\left[M_{A}=11\right.$ frente a $\left.M_{N}=15,58 ; F_{(1,999)}=7,01 ; p=0,00\right]$; en organización de ideas, indicadores lexicales_ $\mathrm{M}_{\mathrm{A}}=4,22$ frente a $\mathrm{M}_{\mathrm{N}}=6,16 ; \mathrm{F}_{(1,999)}=$ $3,66 ; p=0,05]$; en pertinencia $\left[\mathrm{M}_{A}=0,86\right.$ frente a $\mathrm{M}_{N}=0,95 ; F_{(1,899)}=$ 4,38; $p=0,03]$; en hilo argumental $\left[M_{A}=0,45\right.$ frente a $M_{N}=0,76 ; F$, g99. = 11,31; $p=0,00]$; en coherencia total $\left[\mathrm{M}_{\mathrm{A}}=0,76\right.$ frente a $\mathrm{M}_{\mathrm{N}}=$ 2,59; $\left.F_{(1,999)}=35,49 ; p=0,00\right]$; en número de puntos y seguidos $\left[M_{A}\right.$ $=3,45$ frente a $\left.M_{N}=5,73 ; F_{(1,999)}=11,19 ; p=0,00\right]$; en la edad $\left[M_{A}=\right.$ 12,43 frente a $M_{N}=11,7 ; F_{(1,999)}=6,42 ; p=0,01$ ]; y en la nota aportada por el alumno del último trimestre en lengua $\left[M_{A}=3,86\right.$ frente a $M_{N}=$ 5,$\left.59 ; F_{(1,999)}=9,56 ; p=0,00\right]$; así como en la nota aportada por el profesor del último trimestre en lengua $\left[M_{A}=4,62\right.$ frente $a M_{N}=5,89 ; F$ $(1$, g99) $=6,35 ; p=0,01]$.

\section{DISCUSIÓN Y CONCLUSIONES}

Como puede verse, los problemas atencionales son los que más diferencian a los alumnos en base a diferentes medidas de 
composición escrita $u$ otras, lo cual es esperable por lo que sabemos al respecto. Es ampliamente conocido el hecho de que el subtipo inatento es más probable que tenga problemas escolares, mientras que el hiperactivo-impulsivo lo tenga en relación con la conducta (Miranda, Presentación, \& Jarque, 1999; Moreno \& Lora, 2006; Presentación, Miranda, \& Amado, 1999; Vaquerizo-Madrid, EstevezDiaz F, \& Díaz-Maillo, 2006).

Además, el hecho de que la edad, también sea significativa, muestra el posible retraso de estos alumnos en el rendimiento, lo que ya ha sido descrito ampliamente. Ello es confirmado por las diferencias significativas obtenidas en las notas, en estos alumnos frente al resto.

En lo que hace a la prevalencia, los datos indican porcentajes menores a los descritos en los estudios internacionales (Miranda y colaboradores hablan de entre el 3 y el $5 \%$ de los niños en edad escolar) (Garcia, 2002a; Presentación \& Siegenthaler, 2005), si bien al tratarse de alumnos adolescentes sería un resultado esperable, según sugieren los diferentes estudios. El hecho de ser aplicado el cuestionario en el mes de octubre y noviembre, es posible que reflejara un menor conocimiento de los alumnos por parte de los profesores (el curso empieza a en septiembre) que si fuera al final del curso, lo que pudiera haber influido. Así como el hecho de que la colaboración de tantos profesores, no siempre fuera tan entusiasta como la del equipo investigador. En cualquier caso, la ilustración de nuestro estudio evidencia la escasez de estudios existentes, lo que le hace más relevante si cabe.

Además, de la justificación inicial se deduce la necesidad de estudiar el solapamiento, debido a la escasez de estos estudios (Kaplan et al, 2001). Además, se constata la escasez de antecedentes en estos estudios, y cuando se refieren a la escritura son prácticamente inexistentes (Gregg et al, 2002)

Por otra parte, existe la necesidad de intervenciones para mejorar la reflexividad en la composición escrita (Garcia \& de Caso, 2002, 2007), en alumnos con el TDAH. Igualmente, la necesidad de determinar el nivel en la composición escrita y estudiar la naturaleza del solapamiento entre TDAH y composición escrita (Decker, 2001).

Una cuestión de gran interés la plantea con claridad el estudio de Shalev y Tsal (2003) para entender la naturaleza de las dificultades que caracterizan a los niños con déficits atencionales, y se relaciona 
con el papel que juega la edad. Por una parte, los niños más jóvenes tienen mayores dificultades para ajustar el área de la atención; en segundo lugar, la habilidad para centrar la atención en aquello que es relevante e ignorar lo irrelevante que mejora con la edad; y por último, la idea de que los alumnos de menor edad tienen más dificultades para focalizar su atención y localizan de forma más lenta los estimulos relevantes que los de mayor edad. Como resulta que en nuestro estudio se trata más bien de alumnos mayores, ello puede explicar, no sólo una prevalencia menor que en otros estudios con alumnos más pequeños, sino la relación de estos problemas con los niveles de composición escrita, al desatender aspectos esenciales de un texto de calidad como el hilo argumental $u$ otros aspectos de la coherencia textual. En todo caso, parece necesario el desarrollo de otras investigaciones de este tenor. En nuestro caso, desde 1996 venimos desarrollando investigaciones diferentes sobre los procesos y factores intervinientes en la composición escrita y sistemáticamente hemos venido recogiendo los datos percibidos por los profesores y maestros en el cuestionario DSM-IV, y si bien en la mayoría de las investigaciones están analizados, no han sido publicados, representando este artículo una primera presentación y difusión de estos datos que continuará en los próximos meses.

Esta perspectiva se encuentra dentro de una linea de investigación que se viene desarrollando durante los últimos años en la Universidad de León (en evaluación y en intervención), lo que será muy beneficioso tanto para los alumnos con dificultades de aprendizaje como atencionales, asl como para el resto de los alumnos (de Caso \& Garcia, 2006; Garcla, 1999, 2002b; 2007a, 2007b; Garcla, García \& Avias-Gundín, 2004; García \& de Caso, 2004; 2006a, 2006b; Garcla \& Fidalgo, 2003, 2006, en prensa, Garcla \& Marbán, 2003). Pero sobre todo, favorecerá la mejora de la calidad y la productividad de las composiciones escritas de todos los alumnos (Fidalgo, Torrance, \& García, en prensa; Garcla \& Rodríguez, 2007; Torrance, Fidalgo, \& Garcla, 2007), además de los procesos de auto-regulación (Fidalgo \& Garcia, 2008) asl como la reflexividad en general (Garcla \& de Caso, 2007).

Por último, destacar la necesidad de estudiar los procesos implicados en la composición escrita en personas con TDAH (y subtipos), suponiendo esta investigación un intento relevante en este 
sentido intentando confirmar las dificultades de aprendizaje en la escritura como la dificultad escolar más común en el TDAH (IdiazábalAlecha, Guerrero-Gallo, \& Sánchez-Bisbal, 2006), y ello tanto desde la perspectiva epidemiológlca y de evaluación como desde la intervención en composición escrita que contribuirá a su mejora en los alumnos con problemas atencionales, como con hiperactividad e Impulsividad, presenten o no dificultades de aprendizaje o bajo rendimiento $u$ otros problemas educativos o del desarrollo.

\section{REFERENCIAS BIBLIOGRÁFICAS}

Alamargot, D., \& Chanquoy, L. (2001). Through the models of Writing. Kluwer Academic Puvlishers: Dordrecht.

American Psychiatric Association, (1994). Diagnostic and Statistical manual of mental disorders (4 ${ }^{\text {th }}$ ed.) Washington, DC: Author.

Arias, O., \& Garcia, J. N. (2003). Instrumento de revision de la composición escrita (IRCE). XI Congreso de la Psicologia y de la Adolescencia (INFAD). Fuerteventura.

Arias, O., \& Garcla, J. N. (2006). Instrucción en el proceso de revisión textual mediante el procedimierito CDO en estudiantes que presentan bajo rendimiento. Análisis y Modificación de Conducta, 32(145), 693-710.

Arias, O., \& García, J. N. (2007). Eficacia de la instrucción en los aspectos mecánicos y/o semánticos de la revisión textual. Análisis y Modificación de Conducta, 33(147), 5-30.

Barkley, R. A. (1996). Attention-deficit hyperactivity disorder. In: Mash, E.J. and Barkley, R.A. (Eds.), Child psychopatology (pp. 63-112). New York: Guilford Press.

Barkley, R. A. (1997 a). Behavioral inhibition, sustained attention, and executive functions: constructing a unifying theory of ADHD. Psychological Bulletin, 121, 65-94.

Barkley, R. A. (1997 b). ADHD and de nature of self-control. New York: The Guilford Press.

Barkley, R. A. (1998). Definition of ADHD. Joumal of the American Academy of Child \& Adolescent Psychiatry, 37(4).

Barkley, R. A. (2001). Treatment inattentive type of ADHD as a distinct disorder: What remains to be done?. England: Oxford Univ Press. 
Buttler, D., Elaschuk, C., \& Pooles, S. (2000). Promoting Strategic Writing by Postsecundary Students with Learning Disabilities: A Report of Three Case Studies. Learning Disability Quarterly, 23, 196-213.

Cantwell D.P., \& Baker L. (1991). Association Between Attention Deficit-

Hyperactivity Disorder and learning disorders. Joumal of Learning Disabilities, 24(2)

Collings, R. D. (2003). Differences Between ADHD Inattentive and Combined Types on the CPT. Journal of Psychopatology \& Behavioral Assessment, 25(3), 177-189.

de Caso, A. M., \& Garcia, J. N. (2006). What is missing from current writing intervention programmes?. Estudios de Psicologia, 27, 221242.

de la Paz, S. (1999). Composing via Dictation and Speech Recognition

Systems: Compensatory Technology for Students with Learning

Disabilities. Learning Disability Quarterly. 22 (3), 173-182.

Decker S. L., Mcintosh D. E., Kelly A. M., Nicholls S. K., \& Dean R. S. (2001). Comorbidity among individuals classified with attention disorders. Intermational. J. Neuroscience, 110, 43-54.

De la Paz, S. (2001). Stop and Dare: A persuasive writing Strategy. Intervention in School and Clinic, 36(4), 234-243.

Fidalgo, R. \& Garcia, J. N. (Coor.) (2008). Instrucción de la autorregulación y el autoconocimiento (metacognición) en la composición escrita. Barcelona: DaVinci Continental.

Fidalgo, R., Torrance, M., \& García, J. N. (en prensa). The long term effects of strategy-focussed writing instruction for grade six students. Contemporary Educational Psychology.

García, J. N. (Coor.) (1999, 2003, 2005, 2007). Intervención

Psicopedagógica en los Trastomos del Desarrollo. Madrid. Ediciones

Pirámide, $532 \mathrm{pp}$.

García, .J. N. (2002a). Las Dificultades de Aprendizaje y otros Trastornos del Desarrollo. Edupsykhe, Revista de Psicologia y Psicopedagogía, 1(2), 295-312.

Garcia, J. N. (Coor.) (2002b). Aplicaciones de Intervención Psicopedagógica. Madrid. Pirámide.

García, J. N. (Coor.) (2007a). Dificultades del desarrollo: Evaluación e Intervención. Madrid. Pirámide. 
Garcia, J. N. (Coor.) (2007b). Instrumentos de evaluación y programas de intervención en las dificultades del desarrollo. Madrid. Pirámide. (CD) Garcia, J. N., \& Arias-Gundín, O. (2004). Intervención en estrategias de revisión del mensaje escrito. Psicothema, 16 (2), 194-202.

García, J. N., \& de Caso, A.M.(2002). ¿Es posible mejorar la composición en alumnos con dificultades de aprendizaje y/o bajo rendimiento sin que cambie la reflexividad hacia la escritura?. Psicothema. 14(2), 456-462

Garcia, J. N., \& de Caso, A. M. (2006a). Changes in Writing Self-Efficacy and writing Products and Processes through Specific Training in the Self-Efficacy Beliefs of Students with Learning disabilities. Leaming Disabilities, A Contemporary Joumal, 4(2), 1-27.

Garcla, J. N., \& de Caso, A. M. M. (2006b). Comparison of the effects on writing attitudes and writing self-efficacy of three different training programs in students with learning disabilities. International Joumal of Educational Research. 43, 272-289.

Garcia, J. N. \& de Caso, A. M. (2007). Effectiveness of an Improvement Writing Program According to Students' Reflexivity Levels. The Spanish Joumal of Psychology. 10 (2), 303-313.

García, J. N., \& Fidalgo R. (2003). Diferencias en la conciencia de los procesos psicológicos de la escritura: mecánicos frente a sustantivos y otros. Psicothema. 15(1), 41-48.

Garcla, J. N., \& Fidalgo, R. (2006). Effects of two types of self-regulatory instructions programs on students with learning disabilities in writing product, process and self-efficacy. Learning Disability Quarterly, 29 (3), 181-211.

Garcia, J. N. \& Fidalgo, R. (en prensa). The Orchestration of Writing Processes and Writing Products: A comparison of $6^{\text {th }}$ Grade Students With and Without Leaming Disabilities. Leaming Diabilities. A Contemporary Journal.

Garcia, J. N., \& Rodríguez, C. (2007). Influencia del intervalo de registro y del organizador gráfico en el proceso-producto de la escritura y en otras variables psicológicas. Psicothema, 19(2).

Garcia, J. N., \& Sánchez Miguel E. (2001). "The Decade 1989-1998 in Spanish Psychology: An Analysis of Research in Development and Educational Psychology. The Spanish Joumal of Psychology. 4(2), 182-202. 
Graham, S., \& Harris, K. (2000). The role of Self-Regulation and

Transcription Skills in Writing and Writing Development. Educational

Psychologist, 35(1), 3-12.

Graham, S., \& Harris, K. R. (2002). The role of Self-Regulation and

Transcription Skills in Writing and Writing Development. Educational,

Psychologist, 35(1), 3-12.

Gregg, N., Coleman, C., Stennett, R. B., \& Davis, M. (2002). Discourse

Complexity of College Writers With and Without Disabilities: A

Multidimensional Analysis. Journal of Learning Disabilities. 35(1),

23-38.

Idiazábal-Alecha M, Guerrero-Gallo D, \& Sánchez-Bisbal M. M. (2006).

Procesamiento del lenguaje en el trastorno por déficit de atención con hiperactividad. Revista de Neurologia, 42(Sup 2), 29-36.

Kaplan, B. J., Dewey, D. M., Crawford, S. G. \& Wilson, B. N. (2001).

The Term Comorbidity Is Of Questionable Value in Reference to

Developmental Disorders: Data and Theory. Journal of Leaming Disabilities, 34(6), 555-565.

Kellogg, R. T. (1994). The Psychology of Writing. New York, Oxford University. Press.

Knivsberg, A. M., Reichelt, K. L, \& Nodland, M. (1999). Comorbidity or coexistence, between dyslexia and attention deficit hyperactivity disorder. British Joumal of Special Education. 26(1).

Miranda, A., \& Presentación, M. J. (2000). Efectos de un tratamiento cognitivo conductual en nif́os con TDAH, agresivos y no agresivos. Cambio clínicamente significativo. Infancia y Aprendizaje, 91, 5170.

Miranda, A, Presentación, M. J., \& Jarque, S. (1999). La intervención con estudiantes con TDAH: hacia un enfoque contextualizado y multidisciplinar. En J.N. García S., (Coor.), Intervención psicopedagógica en los trastornos del desarrollo (pp. 303-318). Madrid: Pirámide.

Moreno, I., \& Lora, J. A. (2006). Abandonos terapéuticos registrados en el ámbito de la hiperactividad y los trastomos del comportamiento: influencia de variables individuales y familiares. Análisis y modificación de conducta, 32(144), 427-450.

NHIS, (1997-98). Prevalence of Attention Deficit Disorder and Leaming Disability: E.E.U.U. 
Nolan, E. E., Volpe, R. J., Gadow, K. D., \& Sprafkin, J. (1999). Developmental, gender, and comorbidity differences in clinically referred children with ADHD. Journal of Emotional \& Behavioural disorders. 7(1), 11-20.

Presentación, M. J., Miranda, A., \& Amado, L. (1999). Trastorno por déficit de atención con hiperactividad: avances en torno a su conceptualización, bases etiológicas y evaluación. En J.N. García S., (Coor.), Intervención psicopedagógica en los trastornos del desarrollo (pp. 287-302). Madrid: Pirámide.

Presentación, M. J., \& Siegenthaler, R. (2005). Problemática asociada al TDAH subtipo combinado en una muestra escolar. Infancia $y$ Aprendizaje, 28 (3), 261-275.

Tabassam W., \& Grainger J. (2002). Self-concept, attributional style and self-efficacy beliefs of students with learning disabilities with and without attention deficit hyperactivity disorder. Learning Disability Quarterly. 25, 141-151

Taylor, E. (1995). Dysfunctions of Attention. In D. Cincchetti \& D.J. Cohen (Eds.), Developmental psychopathology: Vol. 2. Risk, disorder, and adaptation. New York: Wiley.

Torrance, M., Fidalgo, R., \& Garcla, J. N. (2007). The teachability and effectiveness of strategies for cognitive self-regulation in sixth grade writers. Learning and Instruction. 17(3), 265-285.

Torrance, M., \& Galbraith, D. (Eds.)(1999). Knowing what to write: conceptual processes in text production. Amsterdam: Amsterdam University Press.

Shalev, L., \& Tsal, Y., (2003). The Wide Attentional Window:A Major Deficit of Children with Attention Difficulties. Journal of Learning Disabilities, 36(6), 517-527.

Vaquerizo-Madrid J, Estevez-Diaz F, Dlaz-Maíllo. (2006). Revisión del modelo de alerta e intervención psicolingülstica en el trastomo por déficit de atención e hiperactividad. Revista de Neurologia, 42(Sup 2), 53-61. Willcutt E.G. \& Pennington B.F. (2000). Comorbidity of Reading Disability an Attention-Deficit/Hyperactivity Disorder: Differences by Gender and Subtype. Journal of Learning Disabilities. 33(2), 179-191.

Wong, B. Y. L. (2000). Writing strategies instruction for expository essays for adolescents with and without learning disabilities. Topics in language disorders, 20(4), 29. 